University of Nebraska - Lincoln

DigitalCommons@University of Nebraska - Lincoln

Architectural Engineering -- Faculty Publications

Architectural Engineering and Construction,

Durham School of

3-2006

\title{
Appropriate Characterization of Background Noise Levels in the Workplace
}

\author{
Erica E. Bowden \\ University of Nebraska - Lincoln
}

Lily M. Wang

University of Nebraska - Lincoln, Iwang4@unl.edu

Follow this and additional works at: https://digitalcommons.unl.edu/archengfacpub

Part of the Architectural Engineering Commons

Bowden, Erica E. and Wang, Lily M., "Appropriate Characterization of Background Noise Levels in the Workplace" (2006). Architectural Engineering -- Faculty Publications. 11.

https://digitalcommons.unl.edu/archengfacpub/11

This Article is brought to you for free and open access by the Architectural Engineering and Construction, Durham School of at DigitalCommons@University of Nebraska - Lincoln. It has been accepted for inclusion in Architectural Engineering -- Faculty Publications by an authorized administrator of DigitalCommons@University of Nebraska Lincoln. 


\title{
Appropriate characterization of background noise levels in the workplace
}

\author{
Erica E. Bowden ${ }^{1}$, Lily M. Wang \\ Architectural Engineering Program, University of Nebraska - Lincoln, PKI, $1110 \mathrm{~S}$. \\ $67^{\text {th }}$ St., Omaha, NE 68182, USA \\ ${ }^{1}$ corresponding author: PH (402)554-2074; email: ebowden@mail.unomaha.edu
}

\begin{abstract}
Overall loudness is not the only quality of indoor background noise that affects occupants. The distribution of noise across frequency (pitch), whether or not the noise contains tones, and whether or not the noise changes over time must also be considered. There are several indoor noise criteria systems available to quantify the background noise in rooms, but many of them do not account for these factors. These systems are commonly used by architects and engineers, and often incorporated into manufacturer's data, design guides, and standards. There is much debate over which of the criteria systems best reflect how occupants will respond to noise. To examine these issues, an extensive study is being completed at the University of Nebraska investigating the effects of various types of air-conditioning noise on occupant productivity and perception. Results from performance tests and questionnaires are used to evaluate a number of these noise criteria systems. Specific implications of this project on characterizing noise in the workplace will be discussed.
\end{abstract}

\section{Background}

Noise is generally thought of as "unwanted sound." It can have psychological and physiological effects on people, but individual reactions to a particular noise are very subjective (Kryter 1985). One individual may find a particular noise pleasing, while another person may be quite disturbed by the same sound. Because of the subjective nature of noise perception, quantifying background noise and determining appropriate levels that will satisfy the majority of occupants is a difficult process.

To complicate the issue, overall loudness is not the only quality of background noise that affects occupants. Other properties of noise can also impact one's perception of noise, such as how the noise is distributed across frequency (pitch). For example, does the noise contain a lot of low frequency energy? If so, people might perceive it as sounding "rumbly", or it might even induce vibration in a building. If the noise has a lot of high frequency energy, then it might be described as sounding "hissy." Another commonly used descriptor is "roaring," which means a noise has a lot of middle frequency energy.

A second property of noise that affects occupants is whether or not the noise contains one or more tones, or discrete pitches. Tonal noise is quite commonly 
produced by heating, ventilating, and air-conditioning (HVAC) systems in buildings. For example, motors and fans often exhibit low-frequency pure tones under $350 \mathrm{~Hz}$. Chillers can produce higher frequency tones. There are many more examples of sources that produce tones.

A third property to consider is how the noise behaves over time. Does the noise remain relatively steady or does it fluctuate? If it fluctuates, the change may be rapid (as in a rattling diffuser), or slower (as in a variable air volume (VAV) system that adjusts to changing occupancy).

There are several indoor noise criteria systems available to quantify the background noise in rooms, but many of them do not fully account for these factors. The criteria systems are commonly used by architects and engineers, and often incorporated into manufacturer's data, design guides, and standards. Some of the more commonly used criteria include Noise Criteria (NC; Beranek 1957), Balanced Noise Criteria (NCB; Beranek 1989), Room Criteria (RC; Blazier 1981), Room Criteria Mark II (RC Mark II; Blazier 1997), and A-weighted Equivalent Sound Pressure Level ( $\mathrm{L}_{\mathrm{Aeq}}$; International Organization for Standardization 1987). These criteria provide a single number rating which describes the overall level, or loudness of the sound. Some also include descriptors, such as rumbly, roaring, and hissy, which give an indication of how the background noise will sound to the occupant.

Unfortunately there is some debate over which criteria is the most appropriate to use. Previous studies have shown that there can be large differences among indoor noise criteria systems for the same spectrum (Goodfriend 1975, Tocci 2000, Bowden and Wang 2003). There is no consensus that a particular criterion generally performs better than the others, with preferences among professional societies and consultants varying greatly.

In working environments, the ability of the criteria systems to relate to performance is also of interest. There have been several studies examining the effects of low frequency noise on performance (Landström et al 1991, Holmberg 1993, Persson Waye et al 1997, Persson Waye et al 2001). Some of the conclusions from these studies are that task performance can be affected by noise, the impact can potentially change over time, and the frequency character should be considered. Although this previous work has established that a relationship between noise and performance exists, few have tried to determine whether or not the indoor noise criteria represent this relationship.

To examine these issues, the authors are investigating the effects of various types of air-conditioning noise on occupant productivity and perception. As illustrated in Figure 1, results from performance tests and questionnaires are used to evaluate criteria ratings of $\mathrm{NC}, \mathrm{NCB}, \mathrm{RC}, \mathrm{RC}$ Mark II, and $\mathrm{L}_{\mathrm{Aeq}}$, as well as compared against each other. End results aid in understanding appropriate applications of the various criteria methods. 

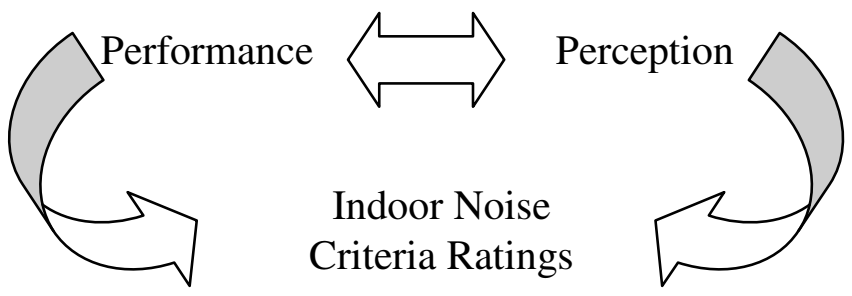

Figure 1 - Three items investigated in the research

\section{General Methodology}

All of the testing is conducted in the "Indoor Environment Lab" at the University of Nebraska. This $25.7 \mathrm{~m}^{3}\left(906 \mathrm{ft}^{3}\right)$ lab resembles a typical office and was specially constructed to be acoustically and thermally controlled. The spaces surrounding the lab remain unoccupied during testing, with the exception of a researcher sitting quietly in an adjacent room. The naturally occurring background noise in the lab is low at $36 \mathrm{~dB} \mathrm{~L}$ Aeq, and the reverberation time is 0.25 seconds at 500 $\mathrm{Hz}$.

The project is being run over three phases. In each phase, subjects are exposed to a wide range of background noise conditions which are typical of HVAC noise situations that might be encountered in offices. The noise conditions are presented over two loudspeakers: an overhead panel speaker and a subwoofer in the corner. The overhead loudspeaker resembles a ceiling tile, and the subwoofer is disguised to resemble an end-table. Under the different noise conditions, subjects complete performance tests and perception questionnaires. Their scores are then related to criteria ratings of the noise.

\section{Phase I: Broadband (non-tonal, non-fluctuating) noise}

Phase I examined the effects of 12 broadband, non-tonal, non-fluctuating background noise conditions. The conditions can be generally categorized as having three levels of loudness (low, medium, and high), and four different spectral qualities (neutral, rumbly, roaring, and hissy). The neutral signals follow a slope of approximately $-5 \mathrm{~dB}$ /octave band. The rumbly, roaring, and hissy signals were obtained by raising the levels in the low, mid, and high frequency ranges by 5-10 dB. Further details on the Phase I noise conditions are available in Bowden and Wang (2005).

Twenty-eight subjects were exposed to each noise condition for approximately 12 minutes. During that time, they spent 90 seconds adapting to the noise, followed by approximately ten minutes of performance testing and then a short questionnaire. Each subject came in for two testing sessions, with each session consisting of one "warm-up trial" followed by six of the 12 noise conditions. Every effort was made to schedule a subject's two test sessions at approximately the same time of day.

Two types of tests were used in this phase: a typing test and a proofreading test. Both tests were administered through a computer program developed by the National Research Council of Canada (Scovil et al 1995a, 1995b). In the typing test, 
subjects were scored for speed and accuracy as they re-typed text presented on a computer monitor. In the proofreading test, subjects compared two columns of 10digit numbers and marked when the columns did not match.

The questionnaire consisted of five questions about the background noise.

Specifically, subjects had to rate their impressions of the loudness, rumble, roar, hiss, and annoyance on seven point discrete scales.

\section{Phase I Results}

Statistical relationships between the three main variables: noise criteria, perception, and performance were analyzed using linear mixed models and Pearson Product Moment Correlations (SPSS 2005, Field and Hole 2003). Additional statistical methods are being considered for future analysis.

Phase I results showed that noise conditions with higher criteria level ratings were perceived as louder, more rumbly, more roaring, and more hissy by test subjects $(p<0.01)$. Figure 2 shows an example, where conditions with higher $\mathrm{L}_{\text {Aeq }}$ levels were perceived as louder. This is the expected trend. Perception of loudness and annoyance were also found to be correlated, as demonstrated in Figure $3(p<0.01)$.

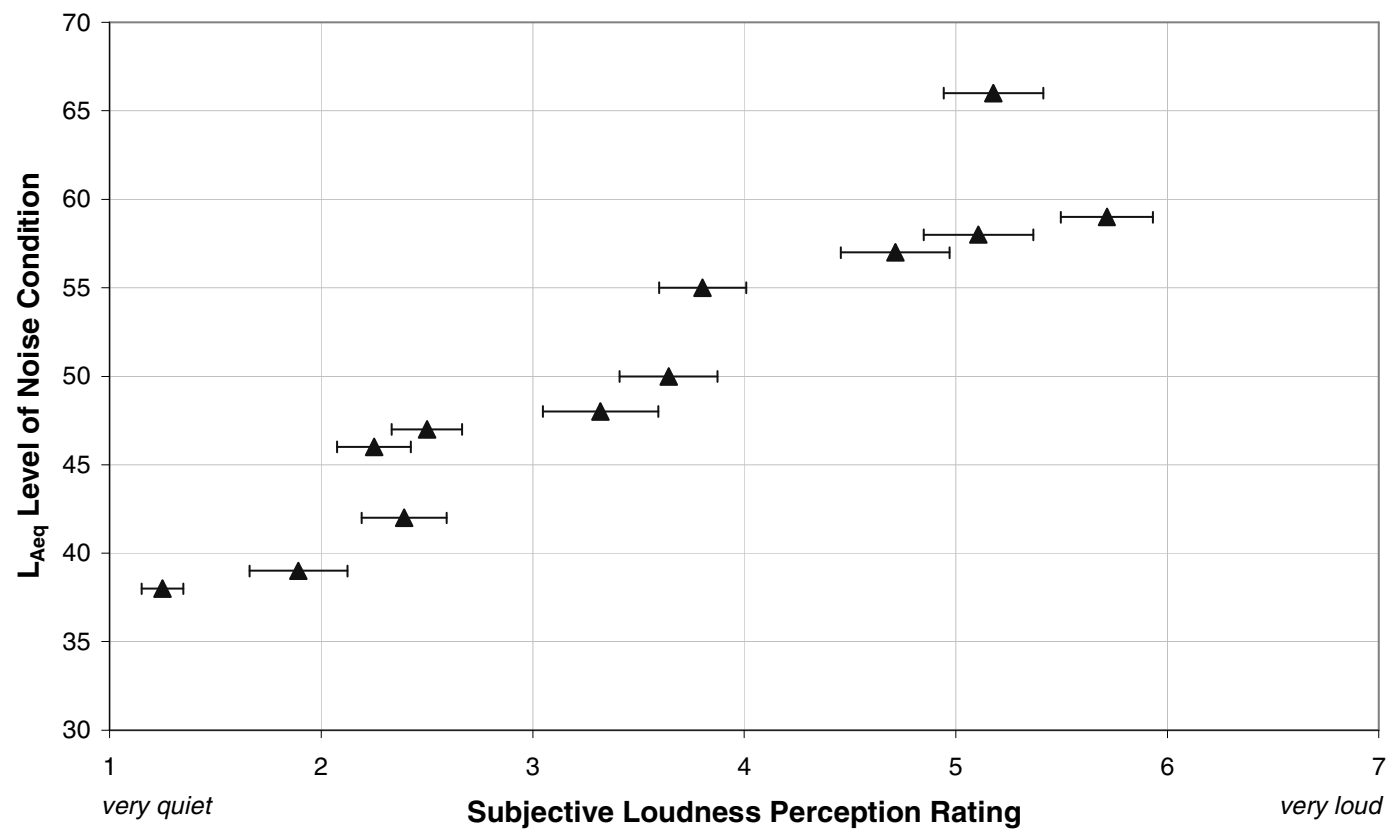

Figure 2 - Phase I subjective loudness perception versus $L_{\text {Aeq }}$ level. Standard error bars are shown. 


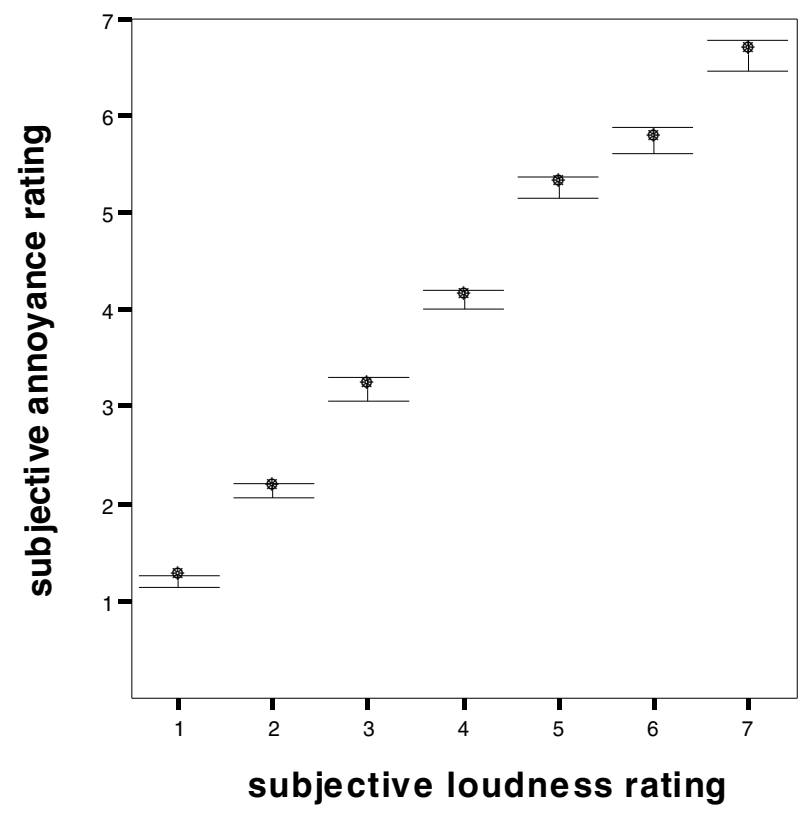

Figure 3 - Phase I subjective loudness ratings versus subjective annoyance ratings averaged across all subjects. Standard error bars are shown. The loudness rating scale ranges from $1=$ very quiet to $7=$ very loud, and the annoyance rating scale ranges from $1=$ not annoying to $7=$ very annoying.

Another aspect of the criteria systems evaluated in this phase was their ability to accurately predict spectral imbalance. That is, the rumbly, roaring, and hissy predictors used by several of the criteria systems were compared to subjective perception. Table 1 shows the different criteria methods analyzed and the spectral quality descriptors they offer. Some of the criteria also provide an assessment of noise induced vibration (NIV), which was not evaluated in this study.

Table 1 - The spectral quality predictors available with the five criteria systems analyzed

\begin{tabular}{|c|c|c|c|}
\hline \multirow{2}{*}{$\begin{array}{c}\text { Criteria } \\
\text { System }\end{array}$} & \multicolumn{3}{|c|}{ Spectral Quality Predictors } \\
\cline { 2 - 4 } & Rumble & Roar & Hiss \\
\hline NC & n/a & n/a & n/a \\
\hline NCB & $\sqrt{ }$ & n/a & $\sqrt{ }$ \\
\hline RC & $\sqrt{ }$ & n/a & $\sqrt{ }$ \\
\hline RC Mark II & $\sqrt{ }$ & $\sqrt{ }$ & $\sqrt{ }$ \\
\hline L $_{\text {Aeq }}$ & n/a & n/a & n/a \\
\hline
\end{tabular}

For this study, RC spectral ratings of rumble agreed with perception $(p<0.01)$, but not necessarily hiss, whereas RC Mark II ratings matched roar $(p<0.01)$ and hiss, but not rumble $(p<0.01)$. NCB spectral ratings of rumble and hiss were not 
significantly related to perception, although the general trend directions were correct. The authors are currently working with a subcommittee of the American Society of Heating, Refrigerating and Air-Conditioning Engineers (ASHRAE) Technical Committee 2.6 on Sound and Vibration regarding possible adjustments to the RC Mark II method to improve these results.

In looking at the performance test results, no significant relationships were observed between the test scores and criteria predictions of level. This may not be surprising as the criteria were developed based on perception surveys, not performance results. However, it was found that typing speed slowed down significantly as subjects perceived the noise as louder or more hissy $(p<0.01)$. Relationships between rumble and roar (low and mid-frequency) perception and performance were less clear.

\section{Intermediate Study: Effects of Exposure Time and Types of Tests Used}

Following Phase I, a study was conducted to look more specifically at the types of tasks used in this research and the time length of exposure to the noise conditions. Further details are presented in Errett et al (2006). To highlight, in Phase I it was found that typing was significantly affected by perception of noise, but no significant results were found with the proofreading tasks. This indicated that the types of tests used can impact the results. Consequently, three types of tests (typing, verbal reasoning, and math) were evaluated in this intermediate study. Results indicate that all three tests may be equally effective in this type of research.

Another area that required more work concerned the appropriate time length of exposure to each noise condition. Would performance on these tasks change if subjects were working under a single noise conditions for 20 minutes versus four hours? General results indicate that performance did not change significantly over time, but that shorter noise exposures (20 minutes) may allow for more back-to-back comparison between sequential noise conditions. Therefore, in on-going work, exposure times of 60 minutes are being used with subjects only exposed to one noise condition per testing day. It is thought that this methodology will more accurately evaluate a subject's "true" feelings about any one noise condition, without influence from the other conditions included in a particular phase. Additional analysis of this intermediate study is currently underway. Specifically, the authors are examining the influence of the types of noise conditions used.

\section{Phase II: Tonal noise}

Results from the intermediate study were used in a Phase II. This portion of the research focuses specifically on discrete tones in noise, how they affect productivity and perception, and how this relates to the indoor noise criteria methods. Six background noise conditions that are controlled to be non-fluctuating over time are being used in this phase:

1. Mid-level neutral condition: broadband noise, simulated with -5 $\mathrm{dB}$ /octave band slope, intersecting $40 \mathrm{~dB}$ at $1000 \mathrm{~Hz}$. This noise condition was also used in Phase I. 
2. Low frequency tonal condition: broadband noise with a low frequency tonal component, measured from an existing facility, with the overall level equalized to $49 \mathrm{~dB} \mathrm{~L}_{\text {Aeq }}$

3. Low frequency tonal condition: similar to condition 2, with the prominence ratio of the tone increased (American National Standards Institute 1995)

4. Mid-frequency tonal condition: broadband noise with a mid-frequency tonal component, measured from an existing facility, with the overall level equalized to $48 \mathrm{~dB} \mathrm{~L}_{\text {Aeq. }}$.

5. Mid-frequency tonal condition: similar to condition 4, with the prominence ratio of the tone increased

6. High frequency tonal condition: broadband noise with a high frequency tonal component, measured from an existing facility, with the overall level equalized to $48 \mathrm{~dB} \mathrm{~L}_{\text {Aeq. }}$.

The general methodology of this phase is similar to Phase I, with a few exceptions. Thirty subjects are exposed to each noise condition for 60 minutes, and hear only one condition per testing day. Under each condition, subjects complete typing, verbal reasoning, and math tests. Further details on the tests are available in Errett et al (2006). The typing tests are very similar to those used in Phase I. The verbal reasoning tests are comprised of true or false questions that deal with the order of letter combinations (Baddeley 1968). The math test consists of simple math statements and short word problems (SkillCheck 2005).

At the end of each testing session, subjects are asked to rate their perception of various qualities of the working environment on a seven point discrete scale. The noise questions ask specifically about loudness, rumble, roar, hiss, tonality, timevariance, distraction, and annoyance. Other questions about the temperature, lighting, and work station comfort are included as well, although the only factor manipulated in the test room is the noise. Following each testing session, the noise is turned off, and subjects are asked to what degree they prefer the current environment as compared to the environment they have been working under for the past hour.

Results from this phase are still under analysis. The subjective performance and perception results are being compared to criteria ratings of the background noise conditions. The impact of the prominence of the tones, as well as the frequency range of the tones (low, mid, and high) are being analyzed as well.

\section{Conclusions}

A variety of indoor noise criteria methods, including NC, NCB, RC, RC Mark II, and $\mathrm{L}_{\mathrm{Aeq}}$ are used by architects, mechanical systems designers, and acousticians to quantify background noise in buildings. There is a lot of debate as to which criteria method is most appropriate to use in the variety of background noise conditions encountered. A series of studies are being conducted at the University of Nebraska to relate several of the more commonly used criteria systems to human performance and perception under HVAC-type noises which might be encountered in working environments. 
In this project, various background noise conditions have been examined over three phases. The noises span a range of sound levels, spectral qualities (neutral, rumbly, roaring, and hissy), and characteristics (broadband, with tones, and with time-fluctuating components). Under each noise condition, subjects complete performance tasks and perception questionnaires. Results show perception of noise can impact performance, but criteria ratings may not reflect this trend. Additionally, it was found that criteria predictions of spectral distribution (rumble, roar, and hiss) did not always match subjective perception. An intermediate study further revealed that performance did not change significantly over time, but that shorter noise exposures (20 minutes) may allow for more back-to-back perception comparison between sequential noise conditions. Research is currently underway to more specifically examine the impact of tones and fluctuations in background noise on perception and performance, and the relationship to indoor noise criteria.

This research provides a good base for how acoustical conditions in offices affect workers, as well as how current criteria methods are related to performance and perception. The impacts this research is having on the fields of acoustical, architectural, and mechanical engineering are very exciting. Even though noise criteria methods are used quite commonly in architectural acoustics and noise control, appropriateness of application remains limited. Based on the results of this work, some refinements to the existing criteria are already under investigation. Ultimately, the goal of any background noise criterion is to accurately reflect occupant response to noise to ensure that acoustical problems in buildings are correctly diagnosed, abated, or prevented. This research brings the field of architectural engineering one step closer to achieving that goal.

\section{Acknowledgements}

This work has been supported by the American Society of Heating, Refrigerating, and Air-Conditioning Engineers (ASHRAE), the Institute of Noise Control Engineering (INCE), and the University of Nebraska - Lincoln Center for Building Integration. The authors are grateful for the assistance of Marc Choiniere, Jessica Errett, and Cathy Novak in administering the subjective tests, and for the recording contributions of Warren Blazier, Norman Broner, Mark Fly, Mark Schaffer, and Jerry Lilly.

\section{References}

American National Standards Institute. (1995). ANSI Standard 1.13-1995: Measurement of Sound Pressure Levels in Air, Acoustical Society of America, Melville, New York.

Baddeley, A.D. (1968). "A 3 min reasoning task based on grammatical transformation." Psychonomic Science, 10, 341-342.

Beranek, L.L. (1957). "Revised criteria for noise in buildings." Noise Control, 3, 1927.

Beranek, L.L. (1989). "Balanced noise-criterion (NCB) curves." J. Acoust. Soc. Amer., 86(2), 650-664. 
Blazier, W.E. (1981). "Revised noise criteria for application in the acoustical design and rating of HVAC Systems." Noise Control Engineering J., 16(2), 64-73.

Blazier, W.E. (1997). "RC Mark II: A refined procedure for rating the noise of heating, ventilating, and air-conditioning (HVAC) systems in buildings." Noise Control Engineering J., 45(6), 243-250.

Bowden, E.E., and Wang, L.M. (2003). "Performance review of indoor noise criteria." Proceedings of the 2003 Architectural Engineering Institute Conference: Building Integration Solutions, Austin, TX.

Bowden, E.E., and Wang, L.M. (2005). "Relating human productivity and annoyance to indoor noise criteria systems: a low frequency analysis." The 2005 ASHRAE Winter Meeting Transactions, Orlando, 111, pt. 1, 684-692.

Errett, J., Bowden, E.E., Choiniere, M., and Wang, L.M. (2006). "Effects of noise on productivity: does performance decrease over time?" Proceedings of the 2006 Architectural Engineering Institute Conference, Omaha, NE.

Field, A., and Hole, G. (2003). How to Design and Report Experiments, Sage Publications, London.

Goodfriend, L. (1975). "A study to update indoor sound criteria for air conditioning systems." RP-126, American Society of Heating, Refrigerating, and AirConditioning Engineers, Inc., Atlanta, GA.

Holmberg, K., Landström, U., and Kjellberg, A. (1993). "Effects of ventilation noise due to frequency characteristic and sound level." J. Low Freq. Noise Vib., 16, $115-122$.

International Organization for Standardization. (1987). ISO Standard 226:1987(E): Acoustics - Normal equal-loudness level contours, International Organization for Standardization, Switzerland.

Kryter, K.D. (1985). The Effects of Noise on Man, Academic Press, Orlando, FL.

Landström, U., Kjellberg, A., Söderberg, L., and Nordström, B. (1991). "The effects of broadband, tonal, and masked ventilation noise on performance, wakefulness and annoyance." J. Low Freq. Noise Vib., 10, 112-122.

Persson Waye, K., Rylander, R., Benton, S., and Leventhall, H.G. (1997). "Effects on performance and work quality due to low frequency ventilation noise." $J$. Sound Vib., 205, 467-474.

Persson Waye, K., Bengtsson, J., Kjellberg, A., and Benton, S. (2001). "Low frequency "noise pollution" interferes with performance." Noise and Health, 4, 33-49.

Scovil, C.Y., Newsham, G.R., and Veitch, J.A. (1995a). "Proofreading Task: Software to Measure the Speed and Accuracy of Proofreading from a Computer Screen.” Internal Report Number 700, Institute for Research in Construction, National Research Council Canada, Ottawa, Canada.

Scovil, C.Y., Newsham, G.R., and Veitch, J.A. (1995b). "Typing Task: Software to Measure the Speed and Accuracy with which Presented Text is Typed."

Internal Report Number 701, Institute for Research in Construction, National Research Council Canada, Ottawa, Canada.

SkillCheck ${ }^{\mathrm{TM}}$ Corporation. (2005). http://www.skillcheck.com.

SPSS Inc. (2005). http://www.spss.com

Tocci, G. (2000). "Room noise criteria - State of the art in the year 2000." Noise/News Intl., 8(3), 106-119. 